\title{
Microplastics: Holistic overview of source, identification, interaction, health and environmental implications and strategies of abatement
}

\author{
Nworie Felix Sunday \\ Department of Industrial Chemistry, Ebonyi State University, PMB 053, Abakaliki, Ebonyi State, Nigeria. \\ *Corresponding Author Email: nworie.Sunday@ebsu.edu.ng
}

\section{DOI: 10.2478/acmy-2021-0004}

\begin{abstract}
:
Microplastic (MP) is currently a subject of discussion in all parts of the world because it has increasingly over the years become the dominant source of pollution in marine ecosystem. Huge number of these Mps emanate from waste management, decommissioning of ships and oil rigs, plastic products in aquaculture and fishery, sewage treatment, consumer products, agricultural production, transportation, offshore oil and gas production and city dust and wears. Microplastic are characteristically non- biodegradable or durable, exhibits buoyancy, travel long distances, complex with toxic chemicals and bioaccumulate being invisible to the human eye. Classification of MPs into primary and secondary based on source and established standard protocols visavis the sampling and identification in matrices were critically reviewed. Physicochemical processes for identification of MPs such as pyrolysis-Gas chromatography/Mass spectrometry, FTIR, Raman spectroscopy, SEM-GS and TGA/MS were reviewed. Deleterious chemicals such as polychlorinated biphenyls (PCBs), polybrominated diphenyl ethers (PBDEs), dioxins, phthalates, polycyclic aromatic hydrocarbons (PAHs) and persistent organic chemicals (POCs) associated with MPs and mechanisms of chelation were appraised. Several menace and health hazards such as hepatic inflammation, genetic mutation, increase in reactive oxygen species (ROS) /oxidative stress, tissue necrosis and proliferation of cells linked with MPs were also discussed. Furthermore, green approaches to arresting the menace such as replacing polymer products with biopolymer an eco-friendly alternative, recycling of plastic products, use of paper bags and glass materials and abrogation of consumer products laced with microbeads were advocated.
\end{abstract}

Keywords: Microplastics; sources; characterization; harmful chemicals; health hazards; green approaches

\subsection{Introduction:}

Production and utilization of plastics globally rose to 348 million metric tonnes in 2017 with an estimated annual increase of $9 \%$ probably because plastics are cheap and can be structured into different applications [1]. The non- biodegradability and bio-accumulative capacity of majority of plastic materials with an estimated $10 \%$ globally end up as waste in the marine environment and $3 \%$ recycled within 2016 year ending [2,3]. Microplastics which represents small debris of plastic materials was first reported by scientist in 1970, but quantitative research into the abundance, detection and effects started early 2004 with increased novel researches in marine and freshwater ecosystems spanning 2013 till date [4]. Much research have been conducted in Europe, Asia, North America and a very few in Africa with high volume of MPs reported in seas, lakes, beaches, rivers and other water bodies [4]. The call for concern of MPs have prompted many researches in several continents with the aim of finding lasting solution to this environmental misnomer [5-10].

Microplastics are described as particles or fragments of plastic less than $5 \mathrm{~mm}$ [11]. Microplastics can be of primary source when they are applied as precursors for the manufacture of industrial goods such as microbeads or as secondary source when they exist as fragments from larger plastic materials. Primary MPs sources include facial cleansers, face wash, skin scrub, hand soap, makeup, shampoo and conditioner, hair dye, sunscreen, baby care products, cleaner, nail polish, deodorant, and toothpaste, cosmetics and microbeads [11]. Secondary MPs include washing of synthetic clothing, wear and tear of tyres, plastic water bottles, car and truck tyre, clothing, cosmetics industry, plastic Manufacturing industries, fishing industry, packaging and shipping, plastic water bottles, sewage treatment plant [4]. Microplastics are present in both surface and underground waters. They can enter the water bodies through runoffs, dumping of plastic wastes, wind, offshore oil and gas production, transportation, sewage treatment and tear and wear of plastic materials, washing of microbeads from cosmetic products and plastic fibres from clothing into waste water treatment plants.

Due to the nature of MPs, handling them remains a problem. The buoyancy, density, and inability of MPs to flocculate makes it impossible to be captured in waste water treatment plants. Microplastics are everywhere, in surface waters, tap waters, sediments, guts and circulatory system of marine organisms; hence the newly coined term, plasticized organism. Microplastics have been shown to be harmful as it blocks the digestive tract, affect reproduction, induce adsorption of pollutants, hamper growth rate, block enzyme production and lower the level of steroid hormone production [12]. Microplastics adsorb persistent organic pollutants and other harmful inorganic compounds because of their high surface area to charge ratio. Ingestion of MPs by aquatic organisms leads to increased toxicity due to accumulation of hydrophobic organic compounds adsorbed to the MPs. Microplastics complex with harmful environmental toxicants such as PAHs, PCBs, PBBs, DDT, organochlorine compounds, dioxins, phthalates and other harmful organic structured compounds and when ingested by animals or inhaled or diffused into the tissue of organism's bio-accumulates and lead to impairment in reproductive cycles, feeding habits, digestion and general physiological disposition of organisms.

Generally, MPs exhibit particle, chemical and microbial toxicity with varying degree of toxicological threats to flora and fauna [10,13-14]. Sampling of Mps in water bodies could effectively be executed using the manta trawl or nueston catamarans for calm and high waves water bodies respectively. Stereomicroscope or mesh of size between 0.05 to $3 \mathrm{~mm}$ helps in size determination while flow meter calculates the concentration of the microplastics. Several authors have employed pyrolysis gas chromatography in combination with mass spectrometry(Pyr-GC-MS), Raman and infrared spectroscopy, thermogravimetric analysis coupled with mass spectrometry(TGA-MS) and scanning electron microscopy in combination with energy dispersive $\mathrm{x}$-ray spectroscopy(SEM-EDs, fluorescence spectrophotometer for the identification of the origin and source of MPs [15].

Microplastics have several beneficiaries which ranges from the producers to consumers and involves cycle of actions. Those who are affected by MPs include the manufacturers, politicians, researchers and households at all levels. In Nigeria, careless and incessant deposition of plastic wastes is common either along the streets or inside water bodies. This normally cause the plastic materials to be washed into the water and some buried deep underground and in both cases debris of MPs are generated unabated. The use of plastic materials for storage and sell of common products such as zobo rod drink, soya milk, kunu and other local beverages in Nigeria calls for serious health concern for the households as the plastic containers are hastily washed and improperly rinsed with untreated water leaving debris of MPs and other contaminants which may be microbe related. Notwithstanding that legislation abounds both locally and internationally on plastics being a pollutant, there has been reckless and unabated mismanagement of plastic wastes especially in Nigeria.

Mandatory monitoring of MPs and standardized monitoring strategy for MPs in EU through the EU Marine Strategy Framework Directive (MSFD-indicator 10.1.3) and Technical sub group on Marine litter has been prescribed [16-19]. The promulgation of National Environmental Regulations which prohibits illicit dropping or deposition of plastic materials on the environment and championed by National Environmental Standards and Regulations Enforcement 
Agency (NESREA) has no strong backing and the implementation dead on arrival. A number of strategies to abate MPs and handle the current misnomer have been advocated and include reduction of plastic waste in the environment, implementing better waste management, reduction in the use of plastic bags, increased awareness of citizens and developing an environmental friendly alternative, The continual unabated accumulation of microplastic in the marine ecosystem calls for a concern [10]. This is imminent in achieving the sustainable development goals with focus on the actualization of clean growth strategy geared towards zero avoidable waste.

Currently there is dearth of research data on MPs stressing on the make-up of polymer materials applying standard operation protocols towards sampling and compositional characterization [19]. Many works on standardization of protocols involving characterization of MPs vary significantly because of different data sources and different methodological approaches [19]. On this premise, the objective of this review is to critically evaluate the different sources, interactions with toxicants, migration and identification of MPs in water samples. The review will also present the health and environmental menace associated with MPs and finally present green alternative approaches that will be adopted for a safer and cleaner environment.

\subsection{Sources of Microplastics}

Microplastics are persistent, non-biodegradable and exhibits buoyancy but can can undergo fragmentation into microsized forms. Microplastics are small fragments of plastics with size less than $5 \mathrm{~mm}$. When the size of MPs is between $20 \mu \mathrm{m}-1 \mathrm{~mm}$ it is regarded as small MPs while when the size ranges from 1-5 mm, it is regarded as large MPs [7]. Microplastics in water bodies differ significantly as they are composed of heterogeneous particles which markedly exhibit variation in size, colour, shape and chemical composition. Pollution of water bodies by MPs emanates from either the sea/other water bodies and land as sources of deposition and consequent mass movement to the oceans [20]. Secondary MPs (evolving from breakdown of manufactured plastic particles by photodegradation through UV radiation or biodegradation (biological processes) or other mechanical processes such as abrasion, wear or tear) and primary MPs (initially made at micro-level sizes for a particular purpose of interest for domestic and industrial applications as in cosmetics, drug delivery systems, textile and clothing and other personal care products) are basically the two main classification of MPs.

The activities that generate MPs are classified into land-based such as untreated or poorly treated municipal sewage, littering of beaches, landfills, industrial leakages, discharges from storm water drains and rivers and floodwaters and sea-based activities such as shipping, illegal deposition at seas, offshore mining and extraction activities, fishing lines, indiscriminately dumped fishing gear [21]. The primary MPs are widely used in the preparation of common household and commercial goods for a given purpose possibly to achieve a desired objective. Some of the products that contain primary MPs include personal care products made with scrub or abrasive lined MPs such as facial cleansers and toothpastes, cosmetics products such as make-up, nail polish, eye shadow, sunscreen, debris from clothing and textile materials, microbeads in cosmetics, industrial accidental plastic debris losses, during-production microplastics debris and discharges from production plants or facilities, drilling fluids for oil and gas exploration, polymeric microspheres for anticancer drug delivery systems $[15,22,23]$. The secondary MPs are products of degradation, fragmentation or weathering of larger plastic products under the influence of high resolved UV radiation, biological degradation or mechanically induced degradation or weathering processes [24]

Plastic materials and bio-based polymers in marine bodies do not undergo complete degradation by biological organisms and therefore constitute serious environmental menace. Other sources of secondary Mps include shipping, abrasion from tyres of cars, lorries and other machines, abrasion of paint during household or industrial use and its exfoliation, offshore and fishing activities which discard plastic materials such as gears and nets into sea bodies releasing MPs which entangles sea animals leading to death and adverse environmental effect [25]. Apart from marine MPs contamination, it has been discovered that millions of persons in the globe drink MPs infested tap water. Current research revealed that MPs are present in tap water and the mean number of fibres per $500 \mathrm{~mL}$ of sampled water in United States, Ecuador, Europe (United Kingdom, Germany, and France), Lebanon, Uganda, India and Indonesia are 94.4, 79.2, 72.2, 93.8, 80.8, 82.4 and 76.2\% respectively [20]. Further investigation into the sources of the MPs revealed that synthetic fabrics are transported through atmospheric deposition into water bodies, rainwater flows carrying MPs into water bodies and employing microplastic filters in water treatment technology which also add to the tap water as they pass through the screens [20].

\subsection{Where and how to get MPs (Sources of MPs)}

Microplastics are everywhere. They are present in the sediments, air, freshwater, marine and drinking water [20]. Laboratory procedures as well as sampling methods lead to increased percentages of MPs due to their ubiquitous nature being able to float freely and travel long distances in air [26]. Sampling of MPs will be considered based on the samples of interest. Water samples: The dimensional sizes of MPs makes it occur in low concentrations in environmental samples and hence large volume of such sample will always be needed for correct identification and characterization processes. Sampling in high wave zones, calm waters and open waters is best done using neuston catamarans, manta trawl and plankton nets respectively [27,28]. Identification of the concentration of MPs in sampling process is important and achieved using flowmeter which calculates the volume of water the net filtered in relation with the concentration of MPs available in the sample. In other words, the concentration of MPs is evaluated by multiplying the horizontal width of net opening with the trawl length. The use of different plankton nets for trawling in the identification of suspended MPs in water columns have been advocated [27].

There are factors that affects trawling and include water currents, weather conditions and trawling time. To ensure that no MPs is left in the trawling process which may invariably affect next operation, the net is usually exteriorly rinsed ejecting the MPs debris and plankton after sample concentration at the cod-end of the net. Sorting of the MPs may be directly done and then kept in the dark or the mixture of debris and plankton stored in a frozen container or in a plastic friendly fixative lined container [7]. The mesh size is relevant in the identification of MPs as the debris collected during sampling and the ones that could be filtered are dependent on the size of the mesh used. Most researchers have used mesh size ranging from 50-3000 $\mu \mathrm{m}$ for sampling MPs [20] The size of the net implying area is a necessary factor for consideration and nets ranging in size between 3-4.5 m with mesh size of about $300 \mu \mathrm{m}$ or less is routinely used for proper sampling of MPs. Sampling of MPs in water is not limited to the use of net. Other sampling methods include screening Continuous Plankton Recorder (CPR) sampling, direct in situ filtration, bulk sampling followed with filtration and most recent direct fractionated pressure developed by $4 \mathrm{H}$-JENA Engineering $\mathrm{GmbH}$ for filtering large volumes of water. Different sizes of MPs are separated through simultaneous fractionation and hypothetically allows size distribution of MPs to as low as $<10 \mu \mathrm{m}[29,30]$.

Sampling in sediments: This involves sampling in beaches or subtidal sampling. Sampling in beaches has some factors such as sample location, the sampling method and the sample depth. The sampling process is not quite difficult and could be achieved using common tools such as shovel, tablespoon or trowel which are non-plastic with a corer or frame for sample identification of location. Even though the sampling process is simple certain consideration has to be given for accurate and replicable results. Authors have reported the sample quantity of between $500 \mathrm{~g}$ to $10 \mathrm{~kg}$, depth size of top $5 \mathrm{~cm}^{3}$ with an extension to $0.3 \mathrm{~m}$ [4]. Sampling of subtidal sediments involves the use of Van Veen or Ekman grab or employing multiple cores. Cores are used for unperturbed surface and depth zones sampling with smaller volumes whereas grabs are for surface and bulk sampling [31]. Standardization of sampling technique is 
still an issue but small (1-5 mm) and large (20 $\mathrm{mm}-1 \mathrm{~mm})$ MPs have been proposed to be sampled using a metal spoon at top $5 \mathrm{~cm}^{3}$ with sediment of about $250 \mathrm{~g}$ [20]. For biota sampling entangled or dead animals such as birds can be collected and dissected and the content of the gut and digestive tract preserved for further analysis through freezing. For animals like earthworm, snails and crabs, the use of net for collection and freezing pending analysis is common whereas bigger animals may be indirectly sampled by collecting their faeces or boluses to study the ingestion of MPs. [32].

\subsection{Laboratory sample preparation}

Microplastics separation from derived sources such as sediments or water or biota components is necessary and easily achieved because of the density difference between the matrices and the microplastic originating source. The density of plastic materials commonly used in our households ranges from $0.05 \mathrm{~g} \mathrm{~cm}^{3}$ for expanded polystyrene to 1.4 and $0.8 \mathrm{~g} \mathrm{~cm}^{3}$ for polyethylene terephthalate (PET) or polyvinyl chloride (PVC) and silicone rubbers respectively [1]. The desired method for the extraction of MPs from sediments with density of $2.65 \mathrm{~g} \mathrm{~cm}^{3}$ involves flotation process in which a saturated solution of salt, say zinc chloride (highly valued for eco-friendliness and cost implication), sodium chloride, sodium iodide and sodium polytungstate solution of high density is mechanically agitated with the dried sample of MPs within a stipulated time. Preferentially, the MPs are stay afloat as suspension while heavier particles settles down. Increase in the concentration of the salt solution (density increase) leads to increase in the MP varieties that can be extracted [29,33]. The employment of different extraction technique determines to a high extent the MPs recovery percentage. The use of these different techniques ranges from the classical laboratory stirring set-up to modified techniques such as elutriation or fluidization coupled with flotation or the use of Microplastic Sediment Separator (MPSS). Generally, the percentage extraction of MPs depends on the shape of plastic debris, size, source and the extraction technique. Newer approaches to MPs recovery give better percentage yield within 68-100 \% whereas the classical approach give yield of 80-100 \% with deficiently extracted small MPs [26, 34].

\subsection{Separation into sizes and purification}

Microplastics samples from water, biota and sediments can be sorted based on size as recently suggested for European Union (EU) monitoring into fractions between $20 \mu \mathrm{m}$ to $1 \mathrm{~mm}$ and 1 to $5 \mathrm{~mm}$. Previously classification was based on MPs less than $500 \mu \mathrm{m}$ and those greater than $500 \mu \mathrm{m}$ [35]. Basically, MPs are sorted and quantified using sieve cascade with mesh size of different sizes when they are of smaller sizes $<500 \mu \mathrm{m}$ whereas when the size is $>500 \mu \mathrm{m}$, manual separation under a stereomicroscope is applied [36]. The purification of MPs is germane and highly relevant for further analysis using specialized instrument. Thus, organic, inorganic and other conjugants need be removed from MPs to prevent result alteration and instrumental damage during identification of MPs. To ensure the result of analysis is not altered, the MPS is either rinsed or equilibrated with clean freshwater, ultrasonically cleaned though with great care to prevent generation of secondary MPs, removal of organic linked impurities with inorganic acids such as $\mathrm{HCl}$ or $\mathrm{HNO}_{3}$, use of $30 \%$ $\mathrm{H}_{2} \mathrm{O}_{2}$ for dried sediment samples, use of sample filter and digestion using hot acid, bases or oxidizing agent [1]. Most recently, there is decline in hot acid or alkaline digestion because some plastics such as polycarbonates and polyamines on interaction with strong acid or alkaline solutions yield other products thereby given preference to sequential enzymatic digestion employing different enzymes such as proteinase, lipase, cellulase, amylase and chitinase which are MPs friendly $[13,26,37]$.

\subsection{Visual identification of MPs}

Visual sorting of Mps from organic and inorganic components is an obligatory and germane step in unmistaken identification of MPs [38]. Small and large MPs are sorted using microscope and visually respectively. Microplastics are sorted based on type, source, shape, color and degradation pattern. To prevent misidentification of MPs, the following criteria was brought up an author [39]. (i) absence of structure of organic origin that is visible in the plastic fibre or particle (ii) exclusion of biologically originated fibres by ensuring thick and three dimensional bending (iii) Presence of clear and homogenously colored plastic debris (iv) Employing fluorescence microscopy for examination of transparent or whitish particles under high magnification to ensure exclusion of substance of biological origin [39]. Visual sorting yields data based on shape, source, degradation pattern and color and the reliability of the data depends on the enumerating focal person, the sensitivity of the microscope and the type of sample. The limitations of visual sorting include high error rate, time unfriendliness and limitation in size to be sorted [28].

\subsection{Chemical characterization of Microplastics}

Chemical characterization of MPs is necessary for proper identification and the commonly used methods are the FTIR, density separation and C.H.N analysis, Raman spectroscopy and pyrolysis GC-MS. The various frequently used methods will be briefly discussed here.

\subsubsection{FTIR Spectroscopy}

Accurate identification of MPs is achieved by the use of fourier transform infrared spectroscopy (FTIR) and for larger MPs attenuated total reflectance (ATR) FTIR. Basically, FTIR gives accurate information on the molecular vibrations of MPs particles and achieved through infrared radiation excitation of sample which is compositional, molecular structural and wavelength dependent [40]. Similarly, the intensity of oxidation of MPs can be exploited in extracting useful information on the physicochemical disintegration of sampled MPs [41]. Specificity of the vibration of the energy of IR at a particular wavelength is a factor that helps in the exact determination of the MPs IR spectra which has been shown to possess distinct bands and highly specific [42, 43]. Recently, some authors have noted that simultaneous evaluation of multiple thousands of spectra within a zone using a singular measurement for extracting chemical images can be achieved using a highly sensitive, high throughput focal plane array- based FTIR imaging [44].

\subsubsection{Density separation and C.H.N analysis}

The density of sampled MPs can be determined by using the specific density of particles to trace the source. Some authors applied this characterization technique by inserting the sample in distilled water and in consideration of the density of the sample a concentrated solution of either strontium chloride, calcium chloride or ethanol was added to ensure buoyancy and density of particle evaluated by determining the weight of a given volume of the solution [38]. The use of C:H:N analyzer with the density measurement helps in determining the elemental composition of MPs and in tracing the origin. For a given polymer material, there is characteristic elemental composition and by comparison, the MPs can be properly assigned to a particular polymer source or treated as a non-polymer. This method is limited by time unfriendliness and small MPs noncompliance due to low throughput.

\subsubsection{Raman Spectroscopy}

One of the commonly used, preferred, straight forward and reliable technique for MPs characterization is Raman spectroscopy [15]. Raman spectroscopy applies surface characterization technique and can be coupled with Raman spectra imaging (microscopy) to give spatial chemical images dependent of Raman spectra of sample or confocal laser-scanning microscopy to detect MPs in biological samples [15]. Subjecting MPs sample to Raman spectra involves irradiation with monochromatic laser beam with wavelength between 500-800 nm which on interaction with atoms and molecules of MPs gives frequency discrepancies between the irradiating laser beam and backscattered light. The difference regarded as Raman shift is evaluated to give particle specific 
Raman spectra which can be compared to the characteristic Raman spectra of polymers.

\subsubsection{Pyrolysis-GC/MS}

The analysis of thermal degradation products of MPs can be achieved by the use of pyrolysis GC/MS which gives the chemical composition. Characteristic pyrograms are obtained on pyrolyzing plastic products and then compared with reference standard, a pyrogram of virgin polymer material [26]. The limitations of this analytical tool include manual insertion of samples in the pyrolysis tube, mono sample analysis per run negating large sample analysis and size unfriendliness as only particles of determined sizes can be manually inserted in the pyrolysis tube [43].

\subsection{Characteristics of microplastics}

The effects of MPs on the environment, man and biota is multifaceted probing several researchers on this area with focus on the some outlined factors. These factors which defines to a great degree the characteristics of Mps includes though not limited to (i) bio-accumulative and non-biodegradable potentials of MPs (ii) buoyancy of MPs which facilitates their presence in marine and freshwater ecosystem with documented data in various world regions (iii) ability to complex by adsorption or absorption with harmful environmental toxicants such as pesticides, PCBs, PBBs and others due to its large surface to volume ratio (iv) Being microsized and light in weight can easily be ingested by marine organisms or easily float or carried through air, thereby entering the food chain leading to several menace (v) its stability (durability) in the environment makes it a suitable carrier of per sistent organic pollutants (POPs) from the environment to organisms $[1,45]$. The consequence of the environmental persistence, buoyancy, non-biodegradability and bio-accumulative potentials of MPs is the increased consumption by man and biota of MPs-metal complex, build of MPs loaded POPs, PCBs, PBDEs, dioxins, tetrabromobisphenol A (TBBPA) and phthalates which leads to alteration or disruption in the physiological status of organisms as shown by pituitary and thyroid hormone imbalance, endocrine disruption, entanglement of animals and infertility in rats [46]. The MPs surface is hydrophobic, crystalline with high surface area to volume ratio and metal cations and organic pollutants binds on the surface of charged MPs through Van Der Waals forces of attraction to form MPs-metal complex [47].

\subsection{Effects of microplastics on the environment, biota and man}

Based on the outlined characteristics of MPs several effects on the environment and biota can be extracted. The physical effects of MPs on marine organisms include entanglement which may lead to death, injuries and blockage of digestive or intestinal pathways, lowering of energy of productive animals, decrease in food consumption due to ingested MPs. There is dearth of data on the effect of Mps on freshwater organisms. Microplastics are hydrophobic with enough exposed surface area to volume ratio endearing the organic environmental toxicants to it through adsorption. Contaminants such as POPs, PCBs, PBDEs, dioxins, tetrabromobisphenol A (TBBPA) and phthalates adsorb to MPs and are carried along as they travel or ingested by organisms. There is observed increase in ingestion of toxicants by aquatic organisms since these pollutants are attached to the MPs in the form of MPs-metal complex desorbed using sodium taurocholate a digestive surfactant [21].

Studies have shown that the desorption of phenanthrene from polyethylene (PE), polypropylene (PP) and polyvinyl chloride (PVC) can be achieved using sodium taurocholate a digestive surfactant [48]. Increased abundance of microorganisms in marine ecosystem could also be associated with the dispersal of microbial species by MPs as they are transported leading to deleterious algal bloom [21]. There is dearth of data on the effect of MPs on the environment but entanglement of animals may lead to death. Humans get exposed to harmful ingested MPs and chemicals when they consume animals infested with MPs-metal complex posing potential risk on the environment as cycle of Mps disposal is formed [49]. Biofilms in drinking water contain loads of microbes which are transported with the water such as tap water causing serious health menace [20]. Studies on the presence of MPs on tap water showed that they are present in tap water between 72.6-94.4 \% laying more credence for the study and its impact on the environment, biota and man [20].

\subsection{Reducing MPs from water bodies}

To reduce MPs in the environment, the following strategies have been advocated as necessary:

(i)Educating the general public on the dangers of illicit plastic deposition in water bodies and land (ii) educating the public on the presence and problems of MPs in consumer products (iii) involving the stakeholders concerned with plastic generation and use such as the manufacturers, politicians, researchers, business owners and households in ensuring effective implementation of Decrees and Acts (iv) devising alternative and better plastic waste management than careless disposal such as recycling (v) bringing up novel ways of waste water treatment, tap water treatment, handling waste from washing machines and acquifers (vi) use of environmentally friendly alternative of polymers such as biopolymers and reusable bags, metal straws and steel containers [1,20].

\subsection{Conclusion}

In conclusion microplastics in the marine, freshwater and air is an evil wind that blows no one any good. Bio-accumulation of MPs in the gut and digestive system of man and biota is increasingly manipulating the mechanism of actions of their bodies altering natural processes and causing deformities. This review outlined the sources, methods of identification, interaction with other environmental pollutants, effects on man and biota and green approaches to arresting the MPs misnomer. The freshwater, marine and even drinking water based on research contains MPs. Many household materials such as clothes, bags generate MPs while MPs are present in some such as facial creams in the form of microbeads. The use of biodegradable materials as replacement of commonly used plastic materials could be a better alternative to MPs. This will reduce health risks such as cases of plasticized animals, and other dangers associated with the ingestion of MPs deleterious loaded chemicals.

\subsection{References}

[1] M.E. Miller, F.J. Kroon, and C.A. Motti, “Recovering microplastics from marine samples: A review of current practices”, Mar. Pollut. Bull., Vol. 123, Pp. 6$18,2017$.

[2] J.R. Jambeck, R. Geyer, C. Wilcox, T.R. Siegler, M. Perryman, and A. Andrady, "Plastic waste inputs from land into the ocean. Science, Vol. 347, Pp. 768$771,2015$.

[3] D.K. Barnes, F. Galgani, and R.C. Thompson, "Accumulation and fragmentation of plastic debris in global environments", Philosophical Transactions of the Royal Society B: Biological Sciences, Vol. 364, Pp. 1985-1998, 2009.

[4] C.M. Rochman, “Microplastics research - from sink to source”, Science, Vol. 360, Pp. 28-29, 2018. doi:10.1126/science.aar7734

[5] K.L. Law, S.E. Morét-Ferguson, D.S. Goodwin, E.R. Zettler, E. Deforce, and T. Kukulka, "Distribution of surface plastic debris in the eastern Pacific Ocean from an 11-year data set", Environ Sci Technol., Vol. 48, Pp. 4732-4738, 2014.

[6] G.A. Burton, "Losing sight of science in the regulatory push to ban microbeads from consumer products and industrial us e", Integr Environ Assess 
Manag., Vol. 11, Pp. 346-34747, 2015.

[7] E. Elena, "Plastic pollution on the Baltic beaches of Kaliningrad region, Russia”, Mar. Pollut. Bull., Vol. 114, No. 2, Pp. 1072-1080, 2017.

[8] J.H. Dekiff, D. Remy, and J. Klasmeier, "Occurrence and spatial distribution of microplastics in sediments from Norderney", Environmental Pollution, Vol. 186, Pp. 248-256, 2014.

[9] J.P.W. Desforges, M. Galbraith, and N. Dangerfield, "Widespread distribution of microplastics in subsurface seawater in the NE Pacific Ocean", Marine Pollution Bulletin., Vol. 79, Pp. 94-99, 2014.

[10] C.E. Enyoh, A.W. Verla and E.N. Verla, “Uptake of Microplastics by Plant: A Reason to Worry or to be Happy?”, World Scientific News, Vol. 131, Pp. 256$267,2019$.

[11] S.L. Wright, R.C. Thompson, and T.S. Galloway, "The physical impacts of microplastics on marine organisms: A review”, Environmental Pollution, Vol. 178, Pp. 483-492, 2013.

[12] T.L. Emma, S.J. Rowland, T.S. Galloway, and R.C. Thompson, "Potential for plastics to transport hydrophobic contaminants", Environmental science \& technology, Vol. 41, Pp. 7759-7764, 2007.

[13] M.A. Cole, "Novel method for preparing microplastic fibers”, Sci. Rep., Vol. 6, Pp. 34519, 2016.

[14] A. Ugolini, G. Ungherese, M. Ciofini, A. Lapucci, and M. Camaiti, “Microplastic debris in sand hoppers”, Estuar Coastal Shelf Sci., Vol. 129, Pp. 19-22, 2013.

[15] M. Cole, H. Webb, and P.K. Lindeque, "Isolation of microplastics in biota-rich seawater samples and marine organisms", Scientific Reports, Vol. 4, Pp. $4528,2014$.

[16] E. Pagter, J. Frias, and R. Nash, “Microplastics in Galway Bay: A comparison of sampling and separation methods”, Mar. Pollut. Bull., Vol. 135, Pp. 932$940,2018$.

[17] M. Oliveira, and M. Almeida, “The why and how of micro(nano)plastic research", TrAC Trends Anal. Chem., Vol. 114, Pp. 196-201, 2019.

[18] D. Schymanski, C. Goldbeck, H.U. Humpf, and P. Furst, "Analysis of microplastics in water by micro-Raman spectroscopy: Release of plastic particles from different packaging into mineral water", Water Res., Vol. 129, Pp. 154-162, 2018.

[19] C. Zarfl, D. Fleet, and E. Fries, “Microplastics in oceans”, Marine Pollution Bulletin, Vol. 62, Pp. 1589-1591, 2011.

[20] S.A. Mason, V.G. Welch, and J. Neratko, “Synthetic Polymer Contamination in Bottled Water", Front. Chem., Vol. 6, Pp. 407, 2018.

[21] M. Kosuth, S.A. Mason, and E.V. Wattenberg, "Anthropogenic contamination of tap water, beer, and sea salt”, PLoS ONE., Vol. 13, Pp. e0194970, 2018.

[22] H.A. Leslie, S.H. Brandsma, and M.J.N. van Velzen, "Microplastics en route: Field measurements in the Dutch river delta and Amsterdam canals, wastewater treatment plants, North Sea sediments and biota", Environment International, Vol. 101, Pp. 133-142, 2017.

[23] J.N. Hitchcock, and S.M. Mitrovic, “Microplastic pollution in estuaries across a gradient of human impact”, Environ. Pollut., Vol. 247, Pp. 457-466, 2019.

[24] M. Wagner, C. Scherer, D. Alvarez-Muñoz, N. Brennholt, X. Bourrain, and S. Buchinger, "Microplastics in freshwater ecosystems: what we know and what we need to know", Environ Sci Europe., Vol. 26, Pp. 12, 2014.

[25] K.J. Wiggin, and E.B. Holland, "Validation and application of cost and time e_ective methods for the detection of 3-500_m sized microplastics in the urban marine and estuarine environments surrounding Long Beach, California", Mar. Pollut. Bull., Vol. 143, Pp. 152-162, 2019.

[26] M.T. Nuelle, J.H. Dekiff, D. Remy, and E. Fries, "A new analytical approach for monitoring microplastics in marine sediments", Environ Pollut., Vol. 184, Pp. 161-169, 2014.

[27] M.J. Doyle, W. Watson, N.M. Bowlin, and S.B. Sheavly, "Plastic particles in coastal pelagic ecosystems of the Northeast Pacific Ocean", Marine Environmental Research, Vol. 71, No. 1, Pp. 41-52.

[28] M. Eriksen, S. Mason, S. Wilson, C. Box, A. Zellers, W. Edwards, H. Farley, and S. Amato, "Microplastic pollution in the surface waters of the laurentian great lakes", Mar. Pollut. Bull., Vol. 77, No. 1e2, Pp. 177e182, 2013.

[29] R.C. Thompson, Y. Olsen, and R.P. Mitchell, “Lost at sea: Where is all the plastic?”, Science, Vol. 304, Pp. 838, 2004.

[30] F. Dubaish, and G. Liebezeit, "Suspended microplastics and black carbon particles in the Jade system, southern North Sea", Water Air and Soil Pollution., Vol. 224, Pp. 1352, 2013.

[31] L. Van Cauwenberghe, and C.R. Janssen, "Microplastics in bivalves cultured for human consumption”, Environmental Pollution, Vol. 193, Pp. 65-70, 2014.

[32] A. Kunz, B.A. Walther, and L. Löwemark, "Distribution and quantity of microplastic on sandy beaches along northern coast of Taiwan”, Marine Pollution Bulletin, Vol. 111, Pp. 126-135, 2016.

[33] M.A. Browne, P. Crump, and S.J. Niven, "Accumulation of microplastic on shorelines worldwide: Sources and sinks", Environmental Science \& Technology, Vol. 45, Pp. 9175-9179, 2011.

[34] H.K. Imhof, R. Sigl, and E. Brauer, "Spatial and temporal variation of macro-, meso- and microplastic abundance on a remote coral island of the Maldives, Indian Ocean", Marine Pollution Bulletin, Vol. 116, Pp. 340-347, 2017.

[35] V. Hidalgo-Ruz, L. Gutow, and R.C. Thompson, "Microplastics in the marine environment: A review of the methods used for identification and quantification", Environmental Science and Technology, Vol. 46, Pp. 3060-3075, 2012. 
[36] K.J. McDermid, and T.L. McMullen, "Quantitative analysis of small-plastic debris on beaches in the Hawaiian archipelago", Marine Pollution Bulletin, Vol. 48, Pp. 790-794, 2004.

[37] A.L. Andrady, “The plastic in microplastics: A review”, Marine Pollution Bulletin, Vol. 119, Pp. 12-22, 2017.

[38] S. Morét-Ferguson, K.L. Law, and G. Proskurowski, "The size, mass, and composition of plastic debris in the western North Atlantic Ocean", Marine Pollution Bulletin, Vol. 60, Pp. 1873-1878, 2010.

[39] F. Norén, L.J. Naustvoll, "Survey of microscopic anthropogenic particles in Skagerrak”, Klima- og forurensningsdirektoratet TA., Vol. 2779-2011, Pp. $1-20,2010$.

[40] K.L. Ng, and J.P. Obbard, "Prevalence of microplastics in Singapore's coastal marine pelagic ecosystems of the Northeast Pacific Ocean", Marine Environmental Research, Vol. 71, No. 1, Pp. 41-52, 2006.

[41] P.L. Corcoran, T. Norris, and T. Ceccanese, "Hidden plastics of Lake Ontario, Canada and their potential preservation in the sediment record", Environmental Pollution, Vol. 204, Pp. 17-25, 2015.

[42] A. Vianello, A. Boldrin, and P. Guerriero, "Microplastic particles in sediments of a lagoon of Venice, Italy: First observations on occurrence, spatial patterns and identification", Estuarine, Coastal and Shelf Science, Vol. 130, Pp. 54-61, 2013.

[43] E. Fries, J.H. Dekiff, J. Willmeyer, M.T. Nuelle, M. Ebert, and D. Remy, "Identification of polymertypes and additives in marine microplastic particles using pyrolysis-GC/MS and scanning electron microscopy", Environ. Sci. Process Impacts, Vol. 15, Pp. 1949-1956, 2013.

[44] I.W. Levin, and R. Bhargava, "Fourier transform infrared vibrational spectroscopic imaging: Integrating microscopy and molecular recognition", Annual Review of Physical Chemistry, Vol. 56, No. 1, Pp. 429-474, 2005.

[45] J. Li, H. Liu, J. Paul Chen, "Microplastics in freshwater systems: a review on occurrence, environmental effects, and methods for microplastics detection", Water Res., Vol. 137, Pp. 362-374, 2018.

[46] N.J. Diepens, and A.A. Koelmans, "Accumulation of plastic debris and associated contaminants in aquatic food webs", Environ. Sci. Technol., Vol. 52, Pp. 8510-8520, 2018.

[47] K. Ashton, L. Holmes, and A. Turner, "Association of metals with plastic production pellets in the marine environment", Marine Pollution Bulletin, Vol. 60, Pp. 2050-2055, 2010.

[48] A. Bakir, S.J. Rowland, and R.C. Thompson, "Competitive sorption of persistent organic pollutants onto microplastics in the marine environment", Marine Pollution Bulletin, Vol. 64, No. 12, Pp. 2782-2789, 2012.

[49] J.P.G.L. Frias, J. Gago, and V. Otero, "Microplastics in coastal sediments from Southern Portuguese shelf waters", Marine Environmental Research, Vol. 114, Pp. 24-30, 2016. 